\title{
Asociación de cultivos, maíz y leguminosas para la conservación de la fertilidad del suelo
}

\author{
ASSOCIATION OF CROPS, CORN AND LEGUMES FOR THE \\ CONSERVATION OF SOIL FERTILITY
}

\author{
SOLEDAD TORRES CALDERON§*, JHON HUARACA FERNANDEZ§, DEYSI LAURA \\ PEZO\$, RENZO CRISÓSTOMO CALDERON§
}

Recibido: 15 junio de 2018 / Aceptado: 20 julio de 2018

§EP. Ingeniería Ambiental, Facultad de Ingeniería y Arquitectura, Universidad Peruana Unión, Perú.

\begin{abstract}
Resumen
La pérdida de fertilidad y la erosión son los problemas más comunes de suelos a nivel mundial, en contraste a ello surgen tecnologías agroecológicas como la asociación de cultivos, que buscan alternativas para el manejo de enfermedades provocadas por plagas, la conservación de la biodiversidad y de los recursos naturales y disminución del riesgo de pérdida de cosechas. Por ello, este artículo tiene como objetivo mostrar y analizar el intercalado de maíz con leguminosas con fines de manejo y conservación de la fertilidad del suelo. Estudios llevados a cabo en varios países demostraron que la asociación de cultivos (policultivo) mejora la eficiencia biológica en comparación con los monocultivos tradicionales. La siembra de policultivos debe tener una distancia adecuada, en caso del maíz $0.9 \mathrm{~m}$ entre hileras por $0.4 \mathrm{~m}$ entre plantas, y de frijoles de $0.9 \mathrm{~m}$ a $0,25 \mathrm{~m}$, que permita que una de las especies libere compuestos alelopáticos que limite la aparición de malas hierbas. Por tanto, esta técnica agroecológica es una alternativa viable para el mejoramiento de la fertilidad de suelos, ya que el intercalado de maíz con leguminosas genera supresión de malezas, conservación de los nutrientes N, P, K del suelo y mejora el rendimiento de grano, incrementando así los beneficios ecológicos y económicos.
\end{abstract}

Palabras clave: alelopáticos, supresión de malezas, intercalado de cultivos y agroecología.

\begin{abstract}
The loss of fertility and erosion are the most common soil problems worldwide, in contrast to this emerge agroecological technologies such as the association of crops, which seek alternatives for the management of diseases caused by pests, the conservation of biodiversity and natural resources and decrease the risk of crop loss. Therefore, this article aims to show and analyze the intercropped corn with legumes for the purpose of management and conservation of soil fertility. Studies carried out in several countries showed that the association of crops (polyculture) improves biological efficiency compared to traditional monocultures. The sowing of polycultures should have an adequate distance, in the case of maize $0.9 \mathrm{~m}$ between rows by $0.4 \mathrm{~m}$ between plants, and beans $0.9 \mathrm{~m}$ to $0.25 \mathrm{~m}$, which allows one of the species to release allelopathic compounds that limits the appearance of weeds. Therefore, this agroecological technique is a viable alternative for the improvement of soil fertility, since the intercrop of corn with legumes generates suppression of weeds, conservation of the nutrients $\mathrm{N}, \mathrm{P}, \mathrm{K}$ of the soil and improves the yield of grain, thus increasing the ecological and economic benefits.
\end{abstract}

Key words: alelopáticos, supresión de malezas, intercalado de cultivos y agroecología

Autor de correspondencia:

Universidad Peruana Unión. Km. 19 Carretera Central, Ñaña, Lima

Tel.: 949621086

E-mail: soledadtorres@upeu.edu.pe 


\section{INTRODUCCIÓN}

Gracias a la agricultura se logra atender la demanda de alimentos de la población, la actividad agrícola ha incrementado en los últimos años generando pero a través del desarrollo de monocultivos, que es desventajosa para la conservación del suelo, por ello surgió la agroecología cuya práctica busca la armonía entre la producción sostenible y conservación del suelo, a través de los policultivos. Ésta promueve la conservación de la biodiversidad y el uso eficiente de los recursos naturales, disminuye el riesgo de pérdida de cosechas y permite mitigar los daños provocados por plagas (Gómez y Zavaleta, 2001).

La agroecología se desarrolla desde la época prehispánica, siendo la asociación del maíz con frijol una técnica ampliamente adoptada en países como Perú donde se desarrolla en terrenos parcelados (Vélez et al., 2007).

En la mayoría de los lugares de producción se practica el monocultivo que tiene desventajas porque contribuye con la disminución de la biodiversidad de los ecosistemas agrícolas, incrementa en la incidencia de plagas y enfermedades en os cultivos, así como la pérdida de fertilidad y erosión de los suelos. En este sistema de cultivo se suele utilizar plaguicidas que incrementan el costo de producción y contamina el ambiental. Por ello, la presente revisión tiene como fin mostrar y analizar la aplicación del intercalado de maíz con leguminosas para el manejo y la conservación de la fertilidad del suelo.

\section{ASOCIACIÓN DE CULTIVOS}

Según Mejía-Zavaleta (1999) la asociación de cultivos (Figura 1) es una forma de policultivo usada por la agricultura tradicional, ecológica y la permacultura; (Muñoz, 2018) afirma que es una de las técnicas más efectivas de la agricultura ecológica, que consiste en plantar dos o más especies en relación de cierta cercanía provocando una relación competitiva y complementaria. Asimismo, Rodríguez et al. (2008) menciona que ésta está basada en la teoría de diferentes cultivos, plantados unos cerca de otros, la que permite un mejor desarrollo por la contribución de nutrientes unos a otros, el control de plagas y malas hierbas, la polinización, etc.

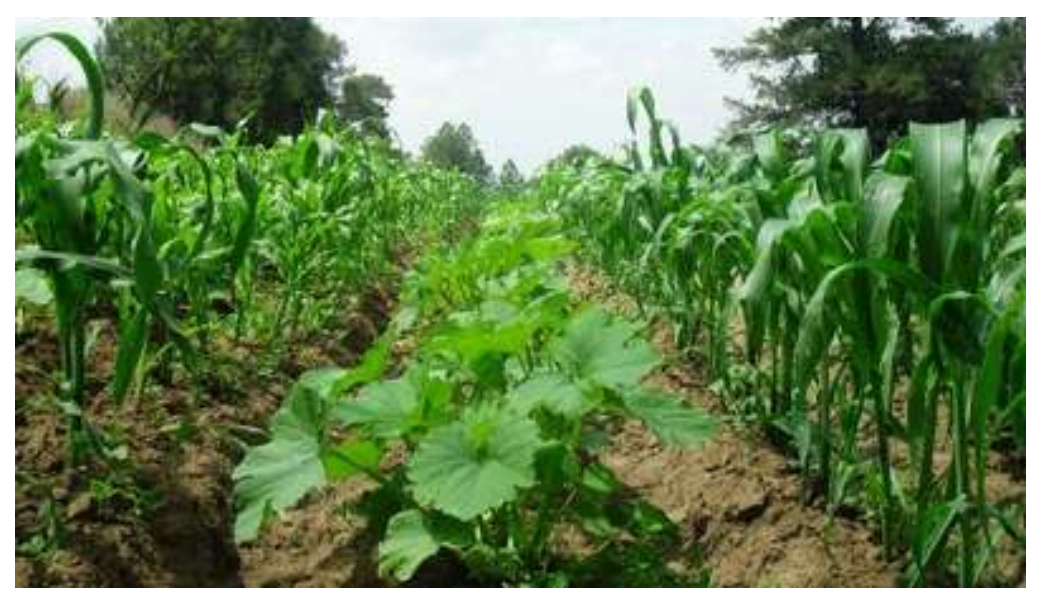

Fig. 1. Asociación de cultivos de maíz con leguminosas. Obtenido del Programa Nacional de manejo, conservación y recuperación de suelos (tomado de: https://viaorganica.org/15451-2/). 


\section{CONSIDERADIONES SOBRE LA TÉCNICA DE ASOCIACIÓN DE CULTIVOS}

\section{MECANISMOS}

Se deben considerar la alternancia de especies de plantas pero buscando que no se vean perjudicadas con enfermedades o plagas, para ello Cabrera (2010) recomienda que los cultivos empleados no deben pertenecer a la misma familia; en este caso el maíz pertenece a la familia de las gramíneas y el frijol a las leguminosas, son de diferentes familias.

Según Guzmán y Mielgo (2008) Existen tres tipos de asociación de cultivos entre la familia de las gramíneas y las leguminosas, y señala como factores de importancia el manejo del espacio y el tiempo, las siguientes:

- Cultivos asociados: se caracteriza por estar constituido de dos o más especies cultivadas en paralelo en parcelas y bajo criterio aleaodio (sin "orden"), no tienen un patrón determinado.

- Cultivos intercalados: están constituidas por dos o más cultivos intercalados en hileras diferentes.

- Cultivos en franjas: en este caso, dos o más especies son cultivadas en simultáneo en distintas franjas de amplitud suficiente para permitir conceder cierto grado de independencia a cada una, pero con suficiente proximidad para su interacción ecológica; ésta es la que permite una mejor mecanización.

\section{DENSIDAD DE SIEMBRA}

Es importante tomar en cuenta la densidad de la siembra ya que según (Castillo, 2016) es tan importante de igual magnitud que un fertilizante, siendo considerado como un insumo más en el proceso de producción; es el número de plantas por unidad de área de terreno que tendrá un marcado efecto sobre la capacidad de producción de las plantas.

\section{CULTIVOS EMPLEADOS}

Uno de los cultivos ampliamente desarrollado es el del maíz; al respecto, es importante tomar en cuenta la densidad de la siembra ya que según (Castillo, 2016) es tan importante de igual magnitud que un fertilizante, siendo considerado como un insumo más en el proceso de producción; es el número de plantas por unidad de área de terreno que tendrá un marcado efecto sobre la capacidad de producción de las plantas.

Del cultivo de leguminosas resaltan los frijoles (Figura 2), según Cabrera (2010) el frijol cuyo nombre científico (Phaseolus) es una leguminosa originaria de América que se cultiva en todo el mundo; constituye una rica fuente de proteínas e hidratos de carbono, además es abundante en vitaminas del complejo B, también proporciona minerales, y presenta un alto contenido de fibra. Existen múltiples variedades de fríjol que se caracterizan por su tamaño, forma, color y tipo de crecimiento. Se considera que en total existen alrededor de 150 especies. Se cultiva en la costa sierra y selva, por ello la amplia adaptabilidad de esta variedad facilita la producción durante todo el año, así mismo mejora los suelos incorporando el nitrógeno atmosférico. 


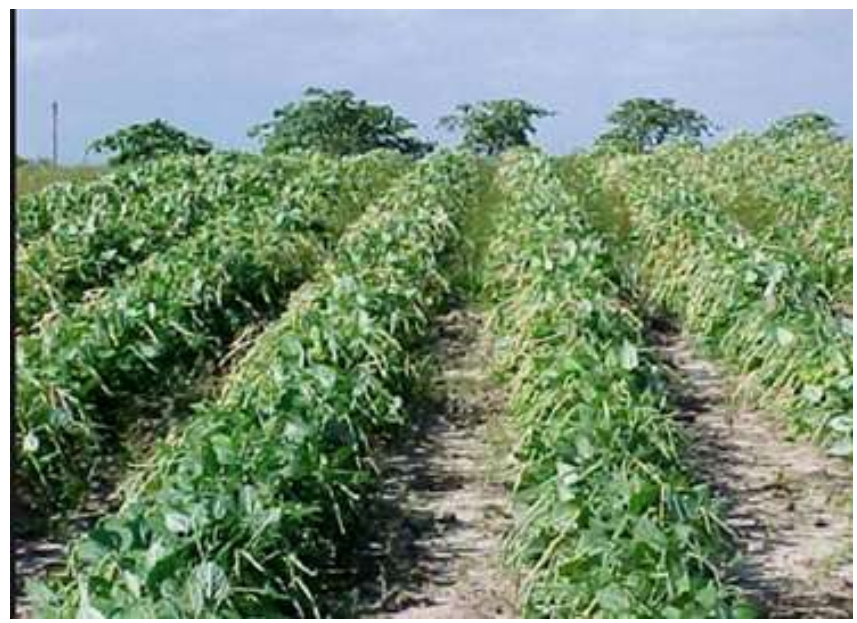

Figura 2. Cultivo de Frijoles. Obtenido de la Producción ecológica y asociación de cultivo (2008)

El maní esta otra especie de cultivo, también muy importante, Según WingChing-Jones y Rojas-Bourrillón (2005) el maní es oriundo de Bolivia o del Noreste de Argentina. Crece en suelos arenosos y franco arenoso, que posea bajo porcentaje de materia orgánica y que tenga una capacidad de intercambio catiónico bajo (3-3,5\%), existe la presencia de $\mathrm{K}$ y $\mathrm{Mg}$ en cantidades medias y profundidad de horizonte A (Johnson et al., 2013).

El cultivo de esta planta en forma perpendicular mejora el control de malezas según (Davis, 2015), el mismo menciona que en una prueba piloto realizada se demostró el control de malezas, que incrementó en un $90 \%$.

\section{MÉTODOS DE CULTIVO}

Según Alfonso et al. (1997), es recomendable que la siembra de policultivos tenga una distancia adecuada en caso del maíz entre $0.90 \mathrm{~m}$ entre hileras x $0.40 \mathrm{~m}$ entre plantas, y en caso de frijoles de $0.90 \mathrm{~m}$ a $0.25 \mathrm{~m}$; asimismo se debe tomar en cuenta la proporción de la fila del intercalado de los policultivos la primera fila de maíz y una de cultivo intercalado de leguminosas con leguminosas, es decir, en una proporción 1:1.

\section{DESARROLLO DE CULTIVOS ASOCIADOS EN EL ENTORNO INTERNACIONAL}

\section{Canada}

La necesidad de aminorar el precio de los insumos agrícolas e incrementar la fertilidad del suelo ha llevado a los investigadores a incursionar en sistemas de producción de cultivos alternativos que incluyen cultivos de fijación de Nitrógeno, siendo el caso de las leguminosas ya que mejoran la calidad del suelo y permiten controlar la erosión y malas hierbas.

Por ello, los experimentos realizados en Canadá con la rotación de cultivos de maíz y leguminosas han demostrado que si se pueden asociar leguminosas y otras plantas como el maíz, la cual ayudará a fijar nitrógeno y a prevenir la erosión de los suelos. En la Tabla 1 se muestran los resultados en distintos lugares de Canadá (McCartney y Fraser, 2010). 
Tabla 1. Producción agrícola en sistema de cultivos asociados

\begin{tabular}{|c|c|c|c|c|c|c|c|c|c|}
\hline Crop/cultivar & Location & Mixture & Rain fed/Irrigation & $\begin{array}{c}\text { Mixture DM } \\
\text { yield (t ha) }\end{array}$ & $\begin{array}{c}\text { Grass species } \\
\text { cut } 1 \mathrm{DM} \\
\text { yieldy } \\
\text { (t ha) }\end{array}$ & $\begin{array}{c}\text { Grass species } \\
\text { cut } 2 \mathrm{DM} \\
\text { yield (t ha) }\end{array}$ & $\begin{array}{c}\text { Legume DM } \\
\text { y ield (t ha) }\end{array}$ & $\begin{array}{l}\text { Total Cypield } \\
\quad(\mathrm{kg} \mathrm{ha})\end{array}$ & Reference \\
\hline \multicolumn{10}{|l|}{ Small-seeded } \\
\hline \multicolumn{10}{|l|}{ Alfalfa } \\
\hline \multicolumn{10}{|l|}{ Medicago sativa $\mathrm{L}$. } \\
\hline Cuf 101 & Kamloops, BC & Barley-ryegrass & 1 & 9.5 & 1.8 & 0.6 & 7 & 1490 & Stout et al.(1997) \\
\hline Nitro & Truro, NS & Barley & $\mathrm{R}$ & 10 & 4.9 & & 5.1 & $905-1170$ & Altinok et al.(1997) \\
\hline \multicolumn{10}{|l|}{ Barrel medic } \\
\hline \multicolumn{10}{|l|}{ Medicago truncatula $\mathrm{L}$. } \\
\hline Borung & Kamloops, BC & Barley-ryegrass & I & 6.2 & 1.8 & 3 & 0.8 & 740 & Stout et al.(1997) \\
\hline Borung & Klamath, OR & Oat & I & 11.6 & & & 0 & 1000 & Dovel and Bohle (1997) \\
\hline Parrabinga & Klamath, OR & Oat & I & 12.3 & & & 0.7 & 1200 & Dovel and Bohle (1997) \\
\hline \multicolumn{10}{|l|}{ Berseem clover } \\
\hline \multicolumn{10}{|c|}{ Trifolium alexandrinum $\mathrm{L}$. } \\
\hline Bigbee & Kamloops, BC & Barley-ryegrass & I & 11.7 & 1.2 & 0.7 & 9.7 & 2110 & Stout et al.(1997) \\
\hline Multicut & Klamath, OR & Oat & I & 11.6 & & & 0 & 1010 & Dovel and Bohle (1997) \\
\hline Bigbee & Edmonton, $\mathrm{AB}$ & Barley & $\mathrm{R}$ & 12.6 & 8.3 & & 4.3 & 1910 & Ross et al. (2004a) \\
\hline Bigbee & Edmonton, $\mathrm{AB}$ & Oat & $\mathrm{R}$ & 12.3 & 8.9 & & 3.4 & 1590 & Ross et al. (2004a) \\
\hline Bigbee & Edmonton, $\mathrm{AB}$ & Triticale & $\mathrm{R}$ & 12.7 & 8.4 & & 4.3 & 1660 & Ross et al. (2004a) \\
\hline \multicolumn{10}{|l|}{ Hairy vetch } \\
\hline \multicolumn{10}{|l|}{ Vicia villosa Roth } \\
\hline Dr.Baumans & Truro, NS & Barley & $\mathrm{R}$ & 11.9 & 1.9 & & 10.9 & $2020-2820$ & Altinok et al. (1997) \\
\hline \multicolumn{10}{|l|}{ Rose clover } \\
\hline \multicolumn{10}{|l|}{ Trifolium pratense $\mathrm{L}$. } \\
\hline Overton & Klamath, OR & Oat & $\mathrm{I}$ & 12.3 & & & 0.5 & 1000 & Dovel and Bohle (1997) \\
\hline \multicolumn{10}{|l|}{ Persian clover } \\
\hline \multicolumn{10}{|c|}{ Trifolium tesupinatum $\mathrm{L}$. } \\
\hline Felix & Kamloops, BC & Barley-ryegrass & I & 9.9 & 6.9 & 0.2 & 8.9 & 1680 & Stout et al.(1997) \\
\hline Felix & Truro, NS & Barley & $\mathrm{R}$ & 9.4 & 4.9 & & 4.6 & $970-1170$ & Altinok et al. (1997) \\
\hline Hamadan & Unarioneown, & Ryegrass & $\mathrm{R}$ & 8.2 & 2.5 & & 5.6 & N.A. & Kunelius and Narasimhalu (1983) \\
\hline \multicolumn{10}{|l|}{ Red clover } \\
\hline \multicolumn{10}{|l|}{ Trifolium pratense $\mathrm{L}$. } \\
\hline Marino & Truro, NS & Barley & $\mathrm{R}$ & 5 & & 6.1 & 11.1 & 820-1980 & Altinok et al. (1997) \\
\hline Khunn & se-Anne-ce- & Corn & $\mathrm{R}$ & 15.4 & & & 0.9 & & Carruthers et al. (2000) \\
\hline Ottawa & 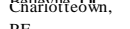 & Ryegrass & $\mathrm{R}$ & 7 & 2.2 & & 4.7 & N.A. & Kunelius and Narasimhalu (1983) \\
\hline
\end{tabular}

Fuente: Obtenido de http://pubs.aic.ca/doi/abs/10.4141/CJPS07182

\section{Chile}

El estudio se realizó en la Estación Experimental Santa Rosa de la Universidad Austral Chile. Se utilizaron cultivos de frejol vista florida (FV), fleetwood (FE), orfeo negro (FO), maíz jubilee (MJ), dulce UACH (MD) y dulce team (MT). Efectuaron quince tratamientos: tres variedades de frijol $(\mathrm{m})$, tres variedades de maíz (n) como cultivo único y nueve parcelas asociadas con todas las combinaciones (Jana et al., 2000)

Tabla 1. Rendimiento en grano seco, valores parciales y totales del índice de área equivalente para frejol y maíz (Tomado de Jana et al. (2000).

\begin{tabular}{|c|c|c|c|c|c|c|}
\hline \multirow[b]{2}{*}{ Tratamiento } & \multicolumn{3}{|c|}{ Rendimiento } & \multicolumn{3}{|c|}{ Rendimiento } \\
\hline & $\begin{array}{c}\text { Frejol } \\
\text { monocultivo }\end{array}$ & $\begin{array}{c}\text { Frejol } \\
\text { asociado }\end{array}$ & $\mathrm{L}_{\text {frejol }}$ & $\begin{array}{c}\text { Maíz } \\
\text { monocultivo }\end{array}$ & Maíz asociado & $\mathrm{L}_{\text {maiz }}$ \\
\hline MJFV & 149,56 & 23,40 & 0,16 & 78,94 & 68,11 & 0,86 \\
\hline MJFF & 155,43 & 25,71 & 0,17 & 78,94 & 70,42 & 0,89 \\
\hline MJFO & 76,51 & 24,95 & 0,33 & 78,94 & 69,01 & 0,87 \\
\hline MDFV & 149,56 & 23,35 & 0,16 & 139,57 & 86,05 & 0,62 \\
\hline MDFF & 155,43 & 26,46 & 0,17 & 139,57 & 77,97 & 0,56 \\
\hline MDFO & 76,56 & 23,27 & 0,30 & 139,57 & 81,12 & 0,58 \\
\hline MTFV & 149,56 & 22,73 & 0,15 & 75,39 & 72,15 & 0,96 \\
\hline MTFF & 155,43 & 18,65 & 0,12 & 75,39 & 70,73 & 0,94 \\
\hline MTFO & 76,51 & 24,11 & 0,32 & 75,39 & 68,51 & 0,91 \\
\hline
\end{tabular}

MJ, MD Y MT = cultivares de maíz Jubilee, Dulce UACH y Dulce. FV, FF, FO = Cultivares de frejol Vista florida, Fleetwood y Orfeo.

Revista de Investigación: Ciencia, Tecnología y Desarrollo (2018) 4(1): 15 - 22 
El tratamiento del frijol demostró que el cultivar orfeo estuvo representado los altos valores ( 0.30 a 0.32$)$, por lo tanto fue el que mejor se adaptó a la asociación por su alta capacidad de competencia y en cuanto al maíz los más altos valores se obtuvieron con el asociación de dulce team seguido por el cultivo jubilee en este caso ambos cultivos presentaron características que les permitieron tener buen desarrollo en condiciones de asociación.

El análisis de varianza evidenció que la relación de área equivalente (LER) en cuatro repeticiones, el sistema de cultivo en asociación de maíz y frijol presentaron mejor ventaja en relación a los cultivos únicos.

\section{VENTAJAS DE ASOCIACIÓN DE POLICULTIVOS}

La asociación de cultivos posee una mayor eficiencia biológica en comparación a los monocultivos, se aprovecha todos los espacios del suelo sembrado a la misma vez, reduce la erosión del suelo por medio de una protección física; además atrae a insectos beneficiosos, entre ellos a los polinizadores o a los que combaten a otros insectos-plaga (Mejía-Zavaleta, 1999).

Aumentan la productividad, de manera que los nutrientes del suelo son mejor aprovechados ya que se integra mayores cantidades de biomasa lo que influye en la productividad de las plantas (González, 2008).

Influencian sobre la población de insectos-plaga, debido a que algunas plantas liberan olores que desagradan a los insectos, a la vez son repelentes y pueden ayudar a controlar las plagas sin necesidad de usar productos químicos (Muñoz, 2014).

Impiden la proliferación de malas hierbas: Debido a una mayor utilización de la superficie se evita el crecimiento de las malas hierbas (Vélez et al., 2007).

Se aprovecha mejor el espacio disponible: si se asocian cultivos que crezcan unos en vertical y otros en horizontal, se aprovecha mucho más la superficie disponible para la siembra generando una rentabilidad económica neta por un mejor uso del suelo, (MejíaZavaleta, 1999).

En la asociación de maíz y frijol, ambas se benefician mutuamente; la similaridad de condiciones de desarrollo, tiempo de siembra y cosecha hace que sean cultivables en asociación. Tanto el maíz como los frijoles requieren de tierra fértil y bien drenada, que haya sido trabajada a seis pulgadas $(15 \mathrm{~cm})$ debajo de la superficie. Ambos demandan alta incidencia solar y pH de aproximadamente 6 (Vélez et al., 2007).

Algunas condiciones favorables en el cultivo de maíz y frijol es que, las plantas de frijol fijan nitrógeno atmosférico en el suelo que el maíz necesita alta y puede utilizar para su desarrollo, puesto que las plantas de frijol no demandan utilizar el nitrógeno que fijan. Los frijoles trepadores se benefician del maíz, ya que pueden torcer los tallos y los utilizan como apoyo para su óptimo crecimiento, así alcanzan con mayor eficiencia la radiación solar (Fernández, 1990).

La biomasa de las malezas se reduce mediante el aumento de la densidad y diversidad de los cultivos (Khan, 2014) 
Un cultivo de legumbre intercalado con maíz es una buena medida para conservar los nutrientes de suelos, ya que estudios experimentales llevados Bengala Occidental según Mandal et al. (2014) evidencia que el estado de fertilidad del suelo después de la cosecha deja una alta disponibilidad de nitrógeno $(\mathrm{N})$ en el suelo, en el cultivo de maíz intercalado con cacahuate, y en el intercalo de policultivos de maíz y maní observaron incremento de potasio $(\mathrm{K})$, sin embargo el $\mathrm{P}$ disponible era bajo esto se debió a la mayor relación de filas. Cuatro filas de cacahuete entre dos filas pareadas de maíz (2:4).

\section{CONCLUSIONES}

La asociación de cultivos de maíz y leguminosas es una técnica agroecológica viable por poseer ventajas como incorporar en el suelo los residuos vegetales que pueden reducir las poblaciones y daños ocasionados por patógenos que atacan a las plantas, favorece la conservación del suelo con el mejoramiento de la fertilidad, mejora el rendimiento del desarrollo de granos en la cosecha de maíz y leguminosas, y permiten beneficios ecológicos y económicos.

\section{Referencias}

Alfonso, C., Riverol, M., Porras, P., Cabrera, E., \& Llanes, J. (1997). Las Asociaciones maíz Leguminosas: Su efecto en la conservación de la fertilidad de los suelos. Agronómica Mesoamericana, 65 -67.

Cabrera, E. (2010). Las asociaciones maíz-leguminosas: su efecto en la conservación de la fertilidad de los suelos. Agronomia Mesoamericana 8(1): 65-73. 1997.

Castillo, J. R. (2016). Densidad de siembra del café variedad en sistemas agroforestales, en el departamento de Santander-Colombia. Cenicafé, 67(1):55-62.

Davis, W. J. (2015). Perpendicular cultivation for improved In-Row Weed Control in Organic Peanut Production. Weed Technology, 128-134.

Fernández, G.Y.M. 1990. Siembra. En: V.C. Liñán, editor, Vademecum del maíz. Semillas Pacífco, Sevilla, ESP. p. 57-128

Gómez Rodríguez, O., \& Zavaleta Mejía, E. (2001). La Asociación de Cultivos una Estrategia más para el Manejo de Enfermedades, en Particular con Tagetes spp. . Revista Mexicana de Fitopatología, 19 (1), 94- 99.

Guzmán G y Mielgo A. (2008). Buenas prácticas en producción ecológica: asociaciones y rotaciones. Ministerio de Medio Ambiente y Medio Rural y Marino. Gobierno de España. Recuperado en 16 de agosto de 2018, de https:/www.mapama.gob.es/es/ ministerio/servicios/publicaciones/Asociaciones\%20y\%20Rotaciones_tcm30-101334.pdf

Johnson, W., Boudreau, M., \& Davis, J. (2013). Combinations of Corn Gluten Meal, Clove Oil, and Sweep Cultivation are Ineffective for Weed Control in Organic Peanut Production. Weed Technology, 27(2), 417-421. Retrieved from http://www.jstor.org/stable/43702068

Khan, S. B. (2014). Effect of intercroping on biomass of weeds and the asssociated Crops. Weed Science, The Univertsity of Agriculture Peshawar Pakistan, 553-562

Mandal M K, Baneerjee M, Banerjee H, Alipatra A and Malik G C. 2014. Productivity of maize (Zea mays) based intercropping system during kharif season under red lateritic tract of West Bengal. International Journal of Life Science 9(1): 31-5.

McCartney, D., \& Fraser, J. (2010). The potential role of annual forage legumes in Canada: A review. Canadian Journal of Plant Science, 90(4), 403-420. http://doi.org/10.4141/CJPS07182

Muñoz, L. (2018). Asociación de cultivo en el Huerto. https://www.agrohuerto.com/asociacion-decultivos-compatibilidad-entre-plantas/ (acceso: 16/08/2018.

Rodríguez González, Horacio, Acosta de la Luz, Lérida, Hechevarría Sosa, Isabel, Milanés

Revista de Investigación: Ciencia, Tecnología y Desarrollo (2018) 4(1): 15 - 22 
Figueredo, Masgloiris, \& Rodríguez Ferradá, Carlos Alberto. (2008). Estudio comparativo entre el monocultivo y la asociación de cultivo en varias plantas medicinales. Revista Cubana de Plantas Medicinales, 13(3) Recuperado en 16 de agosto de 2018, de http://scielo.sld.cu/scielo.php?script=sci_arttext\&pid=S102847962008000300002\&lng=es\&tlng=es.

Vélez Vargas, L., \& Clavijo Porras, J., \& Ligarreto Moreno, G. (2007). Análisis ecofisiológico del cultivo asociado maíz (Zea mays L.) - FRÍJOL VOLUBLE (Phaselus vulgaris L.). Revista Facultad Nacional de Agronomía - Medellín, 60 (2), 3965-3984. Rosa, L. d. (1846). Memoria del cultivo de Maiz. Mexico: Sociedad Literaria.

WingChing-Jones, R., \& Rojas-Bourrillón, A. (2005). Composición nutricional y características fermentativas del ensilaje de maní forrajero. Agronomía Costarricense, 30(1). Recuperado de https://revistas.ucr.ac.cr/index.php/agrocost/article/view/6834

Zavaleta-Mejía, E. (1999). Asociación de cultivo para el manejo de enfermedades. TERRA VOLUMEN 17 NUMERO 3, 1999. 